NMR IN CHEMISTRY 

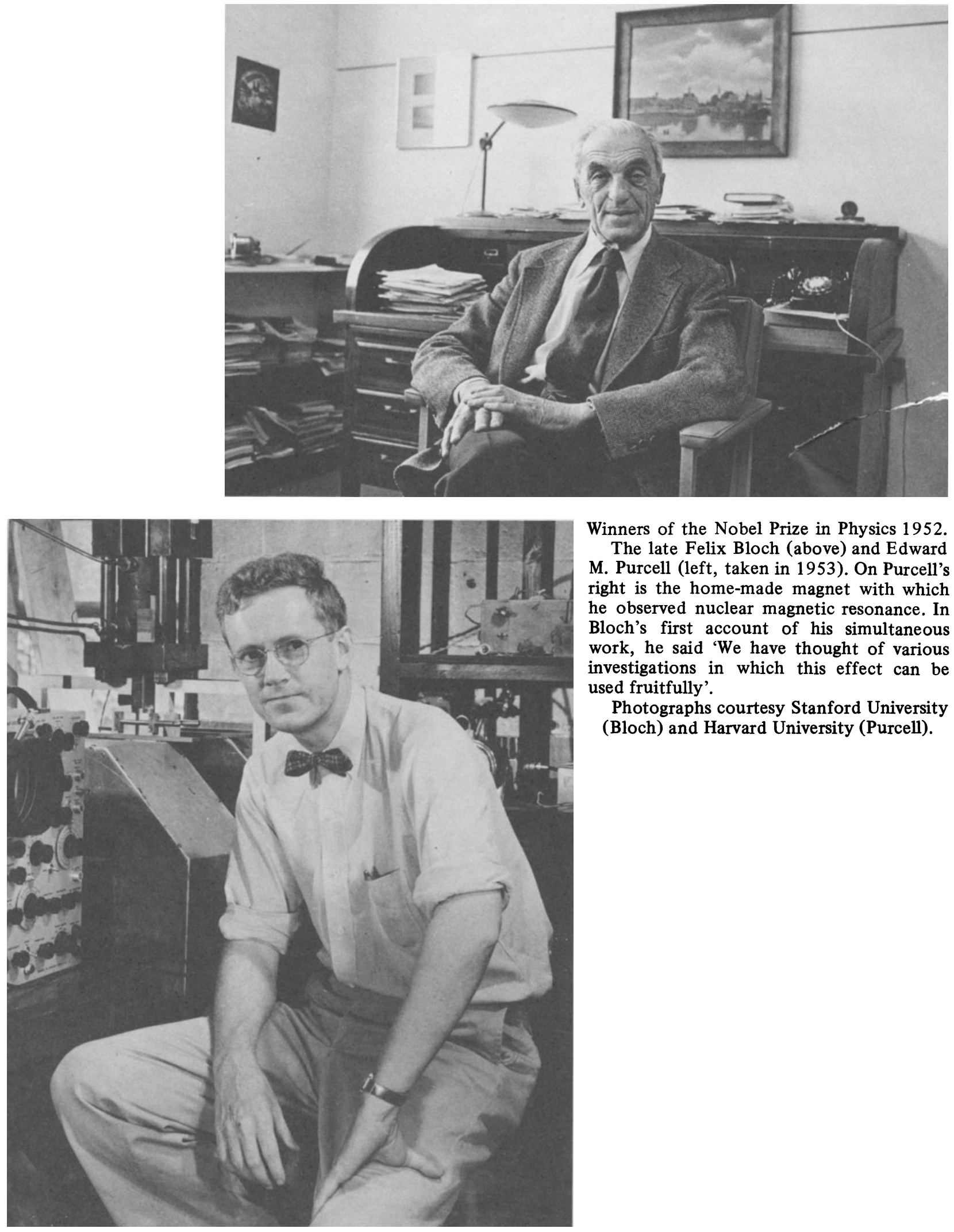

Winners of the Nobel Prize in Physics 1952. The late Felix Bloch (above) and Edward M. Purcell (left, taken in 1953). On Purcell's right is the home-made magnet with which he observed nuclear magnetic resonance. In Bloch's first account of his simultaneous work, he said 'We have thought of various investigations in which this effect can be used fruitfully'.

Photographs courtesy Stanford University (Bloch) and Harvard University (Purcell). 


\section{NMR in Chemistry}

A Multinuclear Introduction

WILLIAM KEMP

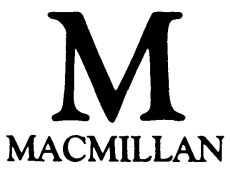


๑ W. Kemp 1986

All rights reserved. No reproduction, copy or transmission of this publication may be made without written permission.

No paragraph of this publication may be reproduced, copied or transmitted save with written permission or in accordance with the provisions of the Copyright Act 1956 (as amended).

Any person who does any unauthorised act in relation to this publication may be liable to criminal prosecution and civil claims for damages.

First published 1986

Published by

MACMILLAN EDUCATION LTD

Houndmills, Basingstoke, Hampshire RG21 2XS

and London

Companies and representatives

throughout the world

Typeset by TecSet Ltd,

Sutton, Surrey

British Library Cataloguing in Publication Data

Kemp, William, 1932-

NMR in chemistry : a multinuclear introduction.

1. Nuclear magnetic resonance spectroscopy

I. Title

541.2 '8

QD96.N8

ISBN 978-0-333-37292-0

ISBN 978-1-349-18348-7 (eBook)

DOI 10.1007/978-1-349-18348-7

\section{To Louie, Again}


Preface

Acknowledgements

$x i$

xiii

1 Introduction to Nuclear Magnetic Resonance NMR

1.1 The Principles of NMR Spectroscopy

1.2 The NMR Spectrometer

1.3 Chemical Shifts

1.4 Field Strength and Frequency

1.5 The Units of Chemical Shift

1.6 Frequency Standards

1.7 Chemical Shift Assignments

1.8 Two Models of Nuclear Magnetic

Resonance

1.8.1 Quantum Mechanics

1.8.2 The Precessing Nucleus

1.9 Coupling and Decoupling of Magnetic Nuclei

2 The Fundamental Basis of Magnetic Resonance 14

2.1 The Spin of Electrons and Nuclei

2.2 Angular Momentum and Magnetic Moments of the Electron and the Proton

2.3 Angular Momentum and Magnetic Moments of Other Nuclei

2.4 The Spin Quantum Number

2.4.1 The Dimension of Spin Angular Momentum

2.4.2 The Direction of Spin Angular Momentum

2.5 Nuclei with Spin Quantum Number $=\frac{1}{2}$

2.6 The Nuclear Magnetic Moment Vector, $\mu$

2.7 The Strengths of Nuclear Magnets - The Nuclear Magneton Unit, $\mu_{\mathrm{N}}$

2.8 The Magnetogyric Ratio $-\gamma$

2.9 Precessional Frequency and $\gamma$ - The Larmor Equation

2.10 Summary of NMR Theory

2.11 Electron Spin Resonance Spectroscopy

2.12 Nuclei with Spin Quantum Number $=1$
2.13 Nuclei with Spin Quantum Number Greater than 1 22

2.14 Quadrupole Moments 23

2.15 Nuclear Quadrupole Resonance Spectroscopy $-\mathrm{NQR}$

2.16 Boltzmann Distributions in NMR - Effects of Field Strength and Temperature 24

2.17 Relaxation Phenomena 25

2.17.1 Spin-Lattice Relaxation and $T_{1} \quad 25$

2.17.2 Spin-Spin Relaxation and $T_{2} \quad 26$

2.18 The Heisenberg Uncertainty Principle and the Width of Absorption Lines

2.18.1 Effect of $T_{1}$ and $T_{2}$ on NMR Line Widths

3 The NMR Spectrometer

3.1 NMR Magnets

3.1.1 Permanent Magnets $\quad 30$

3.1.2 Electromagnets 30

3.1.3 Superconducting Magnets 30

3.1.4 The Search for Bigger Magnets Resolution and Sensitivity

3.1.5 The Direction of $\boldsymbol{B}_{\mathbf{0}}-$ The $z$-axis Convention

3.2 Magnetic Field Homogeneity and Spectrometer Resolution

3.2.1 Shim Coils

3.2.2 Sample Spinning and Spinning Sidebands

Sweep and Frequency Sweep -

Continuous Wave NMR Spectroscopy

3.5 Pulsed Radiofrequency - Fourier Transform NMR Spectroscopy

3.5.1 Fourier Transforms

3.5.2 Advantages of the Pulsed FT NMR Method

3.6 Sensitivity in Multinuclear NMR

3

4

5

6

27

\section{7}

27




\subsubsection{Sensitivity and Nuclear Magnetic Moments}

3.7 Radiofrequency Circuits

36

3.7.1 Radiofrequency Sources in Multinuclear NMR

3.7.2 Single Coil and Crossed Coil Detection

3.7.3 Forced Induction and Free Induction

3.7.4 The Components of RF Signals

3.7.5 Phase Sensitive Detectors and Quadrature Detection

3.7.6 RF Pulses and their Fourier Transforms

3.8 Data Handling in FT NMR

3.8.1 Sampling Rate and Fold-back

3.8.2 The Fast Fourier Transform - Real and Imaginary Solutions

3.8.3 Phases and Filters

3.8.4 Dynamic Range and Computer Word Length

3.9 Variable Temperature Probes

\section{Proton NMR Spectra}

45

4.1 Shielding, Deshielding and Proton Chemical Shifts

4.1.1 Electronegativity and Proton Chemical Shifts

4.1.2 Van der Waals Radii and Proton Chemical Shifts

4.2 Anisotropic Effects and Proton Chemical Shifts

4.2.1 Aromatic Ring Currents

4.2.2 Double Bonds in Alkenes

4.2.3 Carbonyl C=O Double Bonds

4.2.4 Alkyne Triple Bonds

4.2.5 Alkanes

4.3 Chemical and Magnetic Equivalence in

Proton NMR

4.3.1 Free Rotation and Molecular Conformations

4.3.2 Restricted Rotation in Alkenes and Amides

4.3.3 Proton Exchange in Alcohols, Amines, Carboxylic Acids and Keto-Enol Systems

4.3.4 Deuterium Exchange

4.4 Protons Attached to Carbon Atoms Chemical Shift Assignments

4.4.1 Use of Correlation Tables 15.1-15.5
4.4.2 Protons in Alkane Groups - Tables $15.1,15.2$ and 15.3

4.4.3 Protons of Alkene, Alkyne and Aromatic Systems - Tables 15.4 and 15.5

4.5 Protons in $\mathrm{OH}, \mathrm{NH}$ and $\mathrm{SH}$ Groups - Table 15.6

4.5.1 Hydrogen Bonding and Proton Chemical Shifts

4.5.2 Quadrupolar Broadening in NH Signals

4.6 Spin-Spin Coupling - Spin-Spin Splitting $\quad 59$

4.6.1 Predicting Multiplicity from the $(n+1)$ Rule

4.6.2 The Coupling Constant, $J$ - Table 15.7

4.6.3 Theory of Spin-Spin Coupling - I 64

4.6.4 $\mathrm{AX}_{2}, \mathrm{AX}_{3}$, and $\mathrm{A}_{2} \mathrm{X}_{3}$ Coupling Systems

4.6.5 Coupling of Protons to Other Nuclei with Spin $=\frac{1}{2}$

4.7 General Features of Simple Coupling Systems 67

4.7.1 Magnetic Equivalence in Proton NMR

4.7.2 Successive Branching and Pascal's Triangle

4.7.3 Long Range Coupling

4.7.4 Coupling in Proton Exchange Processes

4.9 Non-first Order Spectra

4.10 Simplification of Complex Spectra

4.10.1 Increased Field Strength

4.10.2 Chemical Shift Reagents

4.10.3 Spin Decoupling - Double and Triple Irradiation

4.8.2 Effects of Quadrupole Moments on Observed Splitting

4.11 Additional Problems

5 Carbon-13 NMR Spectra

5.1 Recording Carbon-13 NMR Spectra

5.1.1 Natural Abundance and Isotopomers

5.1.2 Magnetic Moment and Magnetogyric Ratio

5.1.3 Coherent and Broad Band Decoupling $-{ }^{13} \mathrm{C}\left\{{ }^{1} \mathrm{H}\right\}$ NMR Spectra 


\subsubsection{Off Resonance Decoupling}

5.2 Relaxation Effects in Carbon-13 NMR Spectra

5.3 Chemical Shifts in Carbon-13 NMR Spectra

5.3.1 Theory of Carbon-13 Chemical Shifts

5.4 Chemical Shift Assignments in Carbon-13

NMR Spectra

5.4.1 Summarised Procedure - Use of Correlation Tables 15.8-15.16

5.4.2 Identifying the Parent Model

5.4.3 Alkane Chemical Shifts - Table 15.8

5.4.4 Alkene Chemical Shifts - Table 15.9

5.4.5 Alkyne Chemical Shifts - Table 15.10

5.4.6 Aromatic and Heterocyclic Chemical Shifts - Table 15.11

5.4.7 Influence of Functional Substituents on Alkane Chemical Shifts - Table 15.12

5.4.8 Influence of Functional Substituents on Alkene and Aromatic Chemical Shifts - Table 15.13

5.4.9 Carbonyl Group Chemical Shifts Table 15.14

99

101

5.4.10 Miscellaneous Multiple-bonded Carbon Chemical Shifts - Table 15.15

5.4.11 Methyl Group Chemical Shifts Table 15.16

101

101

5.5 Coupling Constants in Carbon-13 NMR

Spectra - Table 15.17 - Satellites

5.5.1 Coupling of Carbon-13 to Hydrogen and Deuterium

5.5.2 Homonuclear Carbon Coupling

5.5.3 Coupling of Carbon-13 to Other Nuclei

5.6 Additional Problems

\section{More Advanced Theories of NMR}

6.1 Theory of Spin-Spin Coupling - II

6.1.1 Electron-coupled Spin-Spin Interactions between Directly-bonded Nuclei

6.1.2 Electron-coupled Spin-Spin Interactions between Protons in $\mathrm{CH}_{2}$ Groups

88

90

90

91

92

93

93

94

95

96

96

97

101

102

102

102

103

108

109

109
6.8

6.8

6.8.1 Longitudinal Relaxation - Spin-

6.8.2 Transverse Relaxation - Spin-Spin

$$
\text { Relaxation }-T_{2}
$$

6.8.3 $T_{2}$ and Field Homogeneity

6.8.4 Line Width, Ringing, $T_{1}$ and $T_{2}$

6.9 The Nuclear Overhauser Effect - NOE

6.9.1 The NOE in Stereochemistry

6.9.2 Theory of the NOE

6.9.3 The Size of the NOE
6.9.4 The Sign of the NOE - Nuclei with Negative $\gamma$

6.9.5 Time Dependence of Spin

Decoupling and the NOE
7 Multipulse and Computational Methods in NMR

7.1 Gated Decoupling and NOE

7.1.1 Coupling, NOE and Time

7.1.2 Gated Decoupling - NOE with Spin Splitting

7.1.3 Inverse Gated Decoupling - Spin Decoupling without NOE

7.1.4 Quantitative Analysis in Decoupled Carbon-13 NMR Spectra 
7.2 Measurement of $T_{1}$ - the Spin-Lattice (or

Longitudinal) Relaxation Time

7.2.1 Inversion Recovery Measurement of $T_{1}$

7.2.2 Other Pulse Sequences for Measuring $T_{1}$

7.2.3 Significance of $T_{1}$ Values

7.2.4 Peak Suppression Methods

7.3 Measurement of $T_{2}$ - the Spin-Spin (or

Transverse) Relaxation Time

7.3.1 Spin Echoes - the Simple Homonuclear Case

7.3.2 Carr-Purcell-Meiboom-Gill (CPMG) Measurement of $T_{2}$

7.3.3 Significance of $T_{2}$ Values

7.3.4 Coupling Constants and Phase Modulation in Spin Echoes

7.4 Polarisation Transfer and Cross Polarisation 7.4.1 Selective Population Inversion

7.5 Summarised Principles of Spin Echoes and Polarisation Transfer

7.6 INEPT, DEPT, and Spectrum Editing by ADEPT

7.6.1 Spectrum Editing - Carbon Subspectra

7.7 Two-dimensional NMR - 2D NMR

7.7.1 J-resolved 2D NMR Spectra

7.7.2 Shift-correlated 2D NMR Spectra

7.7.3 Recording 2D FT NMR Spectra $-\tau$ and the Second Time Domain Axis

7.7.4 ${ }^{13} \mathrm{C}$ Satellite Spectroscopy Carbon-Carbon Connectivity Plots CCCP

7.8 Solid State NMR Spectra

7.8.1 Magic Angle Spinning - MAS

7.8.2 Dipolar Decoupling and Cross Polarisation

\subsubsection{Applications of Solid State NMR}

7.9 Biological NMR

7.9.1 NMR Imaging - Zeugmatography, Tomography

7.9.2 Surface Detection and Topical Magnetic Resonance - TMR

\section{Dynamic NMR}

8.1 Rate Processes and the Heisenberg Uncertainty Principle

8.1.1 Chemical Shift and Exchange Rates 8.1.2 Spin Coupling and Exchange Rates

\subsubsection{Line Shape (Band Shape) and} Exchange Rates

8.1.4 Relaxation Times and Exchange Rates

8.2 Energy of Activation

8.2.1 The Arrhenius Equation and the Eyring Equation

8.2.2 Typical Values of Activation Energies

164

165

8.3 Theory of Coalescence

8.4 The NMR Time Scale - What is it?

8.5 Chemically Induced Dynamic Nuclear

Polarisation - CIDNP

8.5.1 Applications of CIDNP

8.5.2 The Nature of CIDNP

165

166

167

167

168

9 Fluorine-19 NMR Spectra

169

9.1 Fluorine-19 Chemical Shifts

170

9.2 Fluorine-19 Coupling Constants

171

171

9.2.2 Coupling of Fluorine-19 with Proton and Phosphorus-31

9.2.3 Coupling of Fluorine-19 with Carbon-13

9.2.4 Coupling of Fluorine-19 with Nitrogen-14

10 Phosphorus-31 NMR Spectra

10.1 Phosphorus-31 Chemical Shifts

177

10.2

Phosphorus-31 Coupling Constants

10.2.1 Coupling of Phosphorus-31 with Phosphorus-31

177

178

10.2.2 Coupling of Phosphorus-31 with Proton and Fluorine-19

11 Nitrogen-14 and Nitrogen-15 NMR Spectra

11.1 Nitrogen Isotopes $-{ }^{14} \mathrm{~N}$ and ${ }^{15} \mathrm{~N}$

11.1.1 Isotope Abundance

11.1.2 Sensitivity Enhancement - INEPT and DEPT

11.2 Nitrogen Chemical Shifts

11.2.1 Reference Standards for Nitrogen-15 NMR

11.2.2 Factors Influencing Nitrogen-15 Chemical Shifts 
11.2.3 Influence of Protonation and Solvent on Nitrogen-15 Chemical Shifts

185

11.3 Use of Paramagnetic Complexes in Nitrogen-15 NMR

11.4 Nitrogen-15 Coupling Constants

\section{Oxygen-17 NMR Spectra}

12.1 Oxygen-17 Chemical Shifts

12.2 Oxygen-17 Coupling Constants

\section{NMR Spectra from Other Nuclei}

13.1 Deuterium NMR Spectra

13.2 Tritium NMR Spectra

13.3 Boron-11 NMR Spectra

13.4 Aluminium-27 NMR Spectra

13.5 Silicon-29 NMR Spectra

13.6 Selenium-77 NMR Spectra

13.7 Chlorine, Bromine and Iodine NMR Spectra

13.8 Other Elements and their NMR Spectra

\section{Background Physics and Biography of NMR}

14.1 Vector Quantities - Torque and Angular Momentum

14.2 Gyroscopic Behaviour

14.3 Magnetic and Electrical Phenomena

14.3.1 Bar Magnets and Magnetic Moments 14.3.2 Wire Loops and Spinning Charges

14.3.3 Alternating Current

14.3.4 Signal-to-noise Ratios

14.4 Quadrupole Moments

14.5 Historical Retrospects in NMR 14.5.1 Biographical Notes

14.6 Nocturnal Processes

\section{Tables of Reference Data}

210

\section{Proton Data}

15.1 Proton chemical shifts $(\delta)$ for $\mathrm{CH}_{3}, \mathrm{CH}_{2}$ and $\mathrm{CH}$ groups attached to functional group $\mathrm{X}$ 189
15.2 Influence of functional group $\mathrm{X}$ on the chemical shift position $(\delta)$ of $\mathrm{CH}_{3}, \mathrm{CH}_{2}$ and $\mathrm{CH}$ protons $\beta$ to $\mathrm{X}$

15.3 Proton chemical shifts $(\delta)$ for $\mathrm{CH}_{2}$ and $\mathrm{CH}$ groups bearing more than one functional substituent

15.4 Proton chemical shifts $(\delta)$ for protons attached to unsaturated and aromatic groups

15.5 Influence of functional group $\mathrm{X}$ on the chemical shift of protons on benzene rings

15.6 Proton chemical shifts $(\delta)$ for $\mathrm{OH}, \mathrm{NH}$ and SH groups

15.7 Proton-proton coupling constants

\section{Carbon-13 Data}

${ }^{13} \mathrm{C}$ Chemical Shift Summary Chart

$15.8{ }^{13} \mathrm{C}$ Chemical shifts $(\delta)$ in alkane groups

$15.9{ }^{13} \mathrm{C}$ Chemical shifts $(\delta)$ in alkenes

$15.10{ }^{13} \mathrm{C}$ Chemical shifts $(\delta)$ in alkynes

218

$15.11{ }^{13} \mathrm{C}$ Chemical shifts $(\delta)$ in aromatic and heterocyclic molecules

15.12 Influence of functional group $\mathrm{X}$ on the chemical shift of nearby carbons in alkane chains

15.13 Influence of functional group $\mathrm{X}$ on the chemical shift of nearby carbons in alkene

$15.14{ }^{13} \mathrm{C}$ Chemical shifts $(\delta)$ in carbonyl groups

$15.15{ }^{13} \mathrm{C}$ Chemical shifts $(\delta)$ for carbons in various multiple-bonded environments

$15.16{ }^{13} \mathrm{C}$ Chemical shifts $(\delta)$ for methyl groups in common environments

$15.17{ }^{13} \mathrm{C}-{ }^{1} \mathrm{H}$ Coupling constants

$15.18{ }^{13} \mathrm{C}$ Relaxation times $-T_{1}$ (in seconds)

\section{Other Data}

15.19 Solvents used in NMR work

15.20 Greek Alphabet

Further Reading

Answers to Problem Examples

Appendix of Magnetic Isotopes

Compendium of Acronyms in NMR 


\section{Preface}

In the last few years the horizons of nuclear magnetic resonance spectroscopy have all but been pushed out of sight as new and ever more sophisticated instruments are built and reports of new applications flood the literature.

For chemists who are active developers of the science, in whatever aspect, it is difficult merely to read all of the new publications. For chemists who use the method, among other methods, to solve their chemical problems it is well-nigh impossible to keep up to date.

This book is an attempt to introduce NMR to a wide audience of users, in such a cohesive way that all of its potential can be tapped and exploited: it is not a book about the physics and mathematics of NMR, but concerns the interpretation of NMR spectra by those who do not consider themselves particularly mathematical by inclination: the minimum prerequisites are some knowledge of general spectroscopic principles and a familiarity with the chemical properties of common functional classes.

Clear non-mathematical pictures of magnetic resonance must inevitably be distortions of more fundamental laws, but a worthwhile sacrifice is made in forming a bridge between physics and chemical application. The book is obviously not monographic in style or depth, but it will serve its intent if, having read it, the reader is thereby equipped and encouraged to tackle the necessarily more rigorous specialist works.

The emphasis is multinuclear, with a deliberate attempt being made to demystify the NMR studies of the less common NMR elements. Likewise, Fourier transforms and superconducting magnets are introduced very early, and are not given any special status as 'recent developments': continuous wave spectrometers are not referred to as 'conventional', and the use of the terms upfield and downfield is minimised for the same pedagogic reasons (being replaced by lower frequency and higher frequency respectively).

The problem of order of presentation, of finding a beginning, a middle and an end, is not unique to the study of NMR: the chapter sequence adopted should not therefore be taken as a recommended learning programme, but merely as an approximate indicator of the way in which most teachers introduce the subject, building upon the familiar to construct a framework of advanced understanding.

Chapter 1 is set at the most elementary conceptual level, and is an obligatory launching point for the multinuclear approach.

Chapter 4 (on proton NMR) and chapter 5 (on carbon-13 NMR) form the mainstay of the subject; some teachers begin with proton while others begin with carbon-13. Surprisingly little theory is needed to interpret such spectra: for this reason, while chapter 2 is fairly full in explanation, most students will tend to attack this chapter in several short sorties, as the need to know becomes more expansive. The same is true of the instrument details given in chapter 3 which, while fairly detailed and self-contained, can profitably be absorbed in separate parts at different times.

The set of chapters 2, 3, 4 and 5 completes a study of NMR to intermediate level.

Chapters 6 and 7 interrelate with each other at the next most advanced conceptual level: here are introduced the theories and practice made accessible only by microprocessor controlled instrumentation. An argument can be made for introducing some of the chapter 6 theory at an earlier stage, and of course this is not precluded: but there is a strong case to be adduced for consolidating the basic applications in the early chapters before tackling the three-dimensional complexities of the rotating frames of reference, with all the perceptual traps involved.

The study of dynamic molecular processes by NMR is treated separately in chapter 8 . Some of the simpler ideas of dynamic NMR are, however, interspersed in the earlier chapters, since to gather them all in a late chapter would be artificial and distorting.

Chapters 9-13 are all self-sufficient to a degree, and are the barest indications, by example, of the kind of information contained in the NMR spectra of a selection of other nuclei. Given the multinuclear treatment of the earlier chapters, there is no need here for extensive detail.

Chapter 14 very much embodies two personal views. 
Many details of physics are easily forgotten (or may not have been learnt) and having them gathered together serves as a useful aide-memoire for non-physicists. We also often learn nothing about the people who helped to assemble the enormous construct of science, and the sparse biographical notes given here on a few of the famous names in NMR may whet an appetite or two.

Although informative data are supplied as necessary throughout the book, chapter 15 contains a large amount of reference data, which can be accessed for systematic or detailed needs. Extensive tables of chemical shifts, coupling constants and relaxation times are gathered here, with details of common NMR solvents.

There are many problem examples throughout the book. It has been established that one major reason for lack of success in problem solving is an inability to collect and identify only that information which is necessary for solution. In an endeavour to side-step this difficulty, most of the problem examples are preceded by worked examples, so that confidence will rise through knowing which method to apply. Most problems are also seen within the context of the learning objectives under discussion (although other problem examples test more comprehensive understanding).

While it is demonstrably possible to interpret many NMR spectra without intimate study of theory or of spectrometer operation, hopefully the presentation of the subject matter in this book will stimulate an intellectual curiosity, such that a spectrum can be interpreted in the morning, and explained in the afternoon. If the book fulfils its purpose, the user will go back the following morning for more. There is always another experiment to be done.

Heriot-Watt University, Edinburgh

W. K. 


\section{Acknowledgements}

Thanks are due to innumerable people for help and information: for the supply of spectra, or for the permission to reproduce copyright material used in the figures in this book, several companies (and their personnel) have contributed, and this is recognised with gratitude.

The photograph of Felix Bloch shown in the Frontispiece was kindly supplied by Stanford University, California: that of E.M. Purcell was furnished by E. M. Purcell himself.

Bruker Spectrospin published the material in figures 5.3, 5.13, 5.14, 6.12, 7.4, 7.14, 7.16 and 13.2.

Japan Electronic Optical Laboratories, JEOL, supplied the photographs of the spectrometers shown at the head of chapter 3 , and the spectra in figures $1.13,5.1(\mathrm{~b})$, 5.2 (lower), 5.4, 6.14, head of chapter 7 (also used on the book cover) and 11.1.

Oxford Instruments supplied the photograph of the superconducting magnet core and are copyright holders of the diagram showing the cryostat system in figure 3.1.

Oxford Research Systems supplied the photographs of the brain images in figure 7.21 and of the whole-body NMR magnet shown at the head of chapter 10.

Perkin-Elmer published the spectra used in figures $4.18,4.24,8.2,9.2$ and at the head of chapter 6 .

The figures at the head of chapters 8 and 12 were used with the permission, respectively, of the Royal
Society of Chemistry and the American Chemical Society.

Varian Associates granted permission to use the spectra from their NMR Spectra Catalog, namely figures 4.6, $4.7,4.8,4.9,4.10,4.14,4.15,4.20,4.25,4.26,4.27$ and 4.28. Other figures which used spectra published by Varian are figures $5.2,5.11,5.12,7.2,7.5,7.9,7.17$, $10.2,10.4,11.3,11.4,12.2,14.5(\mathrm{c}), 13.1,13.3,13.4$ and 13.5; also those at the heads of chapters 9,11 and 13.

The following were reproduced, with permission, from Kemp, Organic Spectroscopy, 2nd edn, London, Macmillan (1986): 3.2, 4.1, 4.2, 4.3, 4.4, 4.5, 4.12, 4.13, $4.17,4.23,6.7,8.6,14.5(a)$.

Johnson and Jankowsky, Carbon-13 NMR Spectra, New York, Wiley (1972) was the source (with permis: sion) of figures 5.1(a), 5.5, 5.6, 5.7, 5.8, 5.9, 5.10 and 9.4.

Dalton's table of atomic symbols, which appears at the head of chapter 15 , is reproduced by permission of the Trustees of the Science Museum (London).

Many colleagues helped by argument, or by reading part or all of the manuscript (Bill Steedman, Kevin McCullough and Alan Boyd - who also recorded several of the spectra): all are thanked for their encouragement. 